\title{
Clinical significance of microRNA-449a in hepatocellular carcinoma with microarray data mining together with initial bioinformatics analysis
}

\author{
XIA YANG ${ }^{1}$, HAN-LIN WANG ${ }^{2}$, HAI-WEI LIANG ${ }^{1}$, LIANG LIANG ${ }^{3}$, DONG-YUE WEN $^{4}$, \\ RUI ZHANG ${ }^{1}$, GANG CHEN ${ }^{1}$ and DAN-MING WEI ${ }^{1}$
}

Departments of ${ }^{1}$ Pathology and ${ }^{2}$ Cardiology, The First Affiliated Hospital of Guangxi Medical University, Nanning, Guangxi Zhuang Autonomous Region 530021; ${ }^{3}$ Department of General Surgery,

The Second Affiliated Hospital of Guangxi Medical University, Nanning, Guangxi Zhuang Autonomous Region 530007;

${ }^{4}$ Department of Ultrasonography, The First Affiliated Hospital of Guangxi Medical University,

Nanning, Guangxi Zhuang Autonomous Region 530021, P.R. China

Received September 2, 2017; Accepted January 3, 2018

DOI: $10.3892 /$ etm.2018.5836

\begin{abstract}
Increasing evidence has demonstrated that microRNA (miR)-449a expression is reduced in various types of tumors and that it serves as a tumor suppressor. However, the molecular mechanism of miR-449a in hepatocellular carcinoma (HCC) has not been thoroughly elucidated and is disputed. Therefore, the aim of the present work was to systematically review the current literature and to utilize the public Gene Expression Omnibus database to determine the role of miR-449a and its significance in HCC. A total of eight original papers and seven microarrays were included in the present study. Based on the evidence, miR-449a was reduced in HCC. miR-449a is likely involved in various signaling pathways and is targeted to multiple mRNA as part of its function in HCC. In addition, a preliminary bioinformatic analysis was conducted for miR-449a to investigate the novel potential pathways that miR-449a may participate in regarding $\mathrm{HCC}$.
\end{abstract}

\section{Introduction}

The development of high-throughput RNA sequencing technology has led to the discovery of thousands of non-coding RNA (ncRNA) genes $(1,2)$. Until now, the number of newly identified ncRNA genes has been increasing and outnumbers the number of coding transcripts $(3,4)$. Additionally, ncRNAs have been demonstrated to participate in a plethora

Correspondence to: Dr Dan-Ming Wei, Department of Pathology, The First Affiliated Hospital of Guangxi Medical University, 6 Shuangyong Road, Nanning, Guangxi Zhuang Autonomous Region 530021, P.R. China

E-mail: danmingwei08@163.com

Key words: microRNA-449a, hepatocellular carcinoma, gene expression omnibus, bioinformatics analysis of physiological and pathological processes (5-7). Previously, ncRNAs were believed to be the product of random transcription without any intrinsic function $(8,9)$. With the development of ncRNA research, accumulating evidence has unveiled that ncRNAs are functionally important molecules in the cell (5). In general, ncRNAs are a heterogeneous group of molecules and may be categorized into microRNA (miR), long ncRNA (lncRNA), circular RNA (circRNA), endogenous small interfering RNA, Piwi-associated small RNA, small nucleolar RNA (snoRNA), sno-derived RNA, transcription initiation RNA and miR-offset RNA (10). Among them, the function of miR, lncRNA and circRNA have attracted attention from academia. To date, miRs are the most studied ncRNAs, with hundreds of potential target genes, most of which have been bioinformatically predicted (11-13).

miR-449a, located in the first intron of cell division cycle (CDC)20B (chromosome 5q11), belongs to the miR-34/miR-449 families and has been reported to be downregulated in tumors (14). Previous studies also revealed that miR-449a functions as a tumor suppressor by regulating cell proliferation, cycle procession, apoptosis, migration and invasion in multiple human malignances (15-17). As studies focusing on miR-449a and hepatocellular carcinoma (HCC) are currently rather scarce, the role of miR-449a in HCC tumorigenesis remains unclarified.

The incidence of liver cancer is high throughout the world, particularly in East and South-East Asia and Northern and Western Africa (18). Every year, cases in China alone account for $\sim 50 \%$ of the total number of cases and mortalities (19). It has been reported that $\mathrm{HCC}$ is the most common type $(\sim 80 \%)$ of primary liver cancer in the world (18). Currently, with the improvement of hygiene and sanitation, hepatitis $\mathrm{B}$ virus (HBV) and hepatitis $\mathrm{C}$ virus (HCV) infection are decreasing (20). The understanding of the molecular pathogenesis of $\mathrm{HCC}$ has also significantly improved. The incidence of liver cancer is decreasing in some high-risk areas, including China and Japan $(18,20)$. However, HCC remains to have a high incidence rate; therefore, it is urgent that more effective 
diagnostic techniques or replacement therapies are identified for patients with HCC. Collective data from high-throughput analyses of a large number of samples have offered a precise landscape of HCC genetic alterations (21). Indeed, it has enabled the delineation of some key events that may dominate tumor development and progression. It is hoped that translation of this knowledge into targets and biomarkers may impact HCC decision-making and ultimately improve patient outcomes.

The aim of the present work was to systematically review the current literature and to utilize the public Gene Expression Omnibus (GEO) databases to determine the role of miR-449a and its significance in $\mathrm{HCC}$, thereby helping to understand and explore the pathogenesis of miR-449a in HCC. In addition, a preliminary bioinformatics analysis was conducted for miR-449a to investigate the novel potential pathways that miR-449a may participate in.

\section{Materials and methods}

Literature retrieval. To identify the role of miR-449a in HCC, a systematic literature search was conducted in the electronic databases PubMed (https://www.ncbi.nlm.nih.gov/pubmed), Embase (https://www.embase.com/), Web of Science (https://apps.webofknowledge.com/), China Biology Medicine disc (CBMdisc) (http://www.sinomed.ac.cn/zh/), China National Knowledge Infrastructure (CNKI) (http://www. cnki.net/), WANFANG DATA (http://g.wanfangdata.com. $\mathrm{cn} /$ ), Chongqing VIP (http://qikan.cqvip.com/) and Google Scholar (https://scholar.google.com) until October 2017. The key words were as follows: 'Malignan* OR cancer OR tumor OR tumor OR neoplas* OR carcinoma,' 'hepatocellular OR liver OR hepatic OR HCC' and 'miR-449a OR miRNA-449a OR microRNA-449a OR miR 449a OR miRNA 449a OR microRNA 449a.' The articles were selected for the study if they met the following criteria: i) Studies published in Chinese or English; ii) studies assessing the dysregulation of miR-449a in $\mathrm{HCC}$; iii) the expression profiles of miR-449a in patients with HCC and healthy controls were available; and iv) studies conducted on human tissues or body fluids, such as serum and urine. A total of 9 papers from PubMed, 5 from Google Scholar and 19 from the Web of Science were identified in the initial search, while only one study was found in the four Chinese databases (CBMdisc, CNKI, WANFANG DATA and Chongqing VIP). Following the elimination of duplicate studies, eight studies met the inclusion criteria. Review articles were not included in the present study. In the second step, the references and related citations of the eight articles were checked for additional information.

GEO microarray chip screening. To guarantee more comprehensive data collection, the GEO database of the National Center for Biotechnology Information of the National Institute of Health of USA (http://ncbi.nlm.nih.gov/geo/) was searched, which is a public functional genomics data repository. The following terms were used: 'Malignan* OR cancer OR tumor OR tumor OR neoplas* OR carcinoma' AND 'hepatocellular OR liver OR hepatic OR HCC.' Eligible microarrays were downloaded to further extract the miR-449a expression values, which contained two sides of the miR-449a expression level
(HCC and normal control). In each dataset, the mean value and standard deviation were calculated. Following this, all datasets were gathered for the assessment of miR-449a expression in HCC with STATA 12.0 (StataCorp LLC, College Station, TX, USA). The standardized mean difference (SMD) and $95 \%$ confidence interval (CI) were determined for the pooled values. As the SMD is the mean difference (MD) divided by the standard deviation (SD) and MD is the mean difference of the tumor group and the control group (22), $\mathrm{SMD}=0$ meant that there was no significant difference of miR-449a expression in HCC tissues and normal control, $\mathrm{SMD}<0$ means that miR-449a was downregulated in HCC and SMD $>0$ means that miR-449a was upregulated in HCC. A sensitivity analysis was also conducted with STATA 12.0 'metainf' command line (23).

Screening differentially expressed genes and predicting target genes. GEO Profiles (https://www.ncbi.nlm.nih. gov/geoprofiles/) was searched to explore the differentially expressed genes associated with miR-449a in HCC using the terms 'HCC AND miR-449a.' Finally, one dataset (GSE74710) (24) demonstrated that the transient transfection of miR-449a mimics into an HCC cell line, HLE, identified putative target genes of miR-449a. The dataset was downloaded to calculate the fold change (FC) between the group transfected with the miR-449a mimics and the negative control by R/Bioconductor (version 3.4.2, https://www.R-project.org/) $\mathrm{FC}<0.5$ and $\mathrm{P}<0.05$ were selected.

On the other hand, target genes of miR-449a were predicted using miRWalk 2.0 (http://zmf.umm.uni-heidelberg. de/apps/zmf/mirwalk2/) (25), which combined 12 existing miR-target prediction programs (miRWalk, Microt4, miRanda, mirbridge, miRDB, miRMap, miRNAMap, Pictar2, PITA, RNA22, RNAhybrid and TargetScan) to provide comprehensive potential targets for miR-449a. The genes predicted in more than five prediction software programs were regarded as reliable targets of miR-449a.

In addition, a novel computerized approach, Natural Language Processing (NLP), was performed to obtain all the genes related to HCC (details have been described previously) (26). Finally, three components that were overlapped were identified for further bioinformatics analysis.

Functional and signaling pathway analysis. The functional and signaling pathway analyses of the selected genes were performed on a public database platform, the Database for Annotation, Visualization and Integrated Discovery (DAVID) (http://david.ncifcrf.gov/). The analyses included Gene Ontology (GO) function analysis (http://geneontology.org/) and Kyoto Encyclopedia of Genes and Genomes (KEGG) (http://www.genome.jp/kegg/) analysis. The GO function analysis included three categories, namely, the biological processes (BP), cellular components (CC) and molecular functions $(\mathrm{MF})$. The results of the $\mathrm{GO}$ analysis were visualized as co-expression networks via Cytoscape v3.4.0 (http:// cytoscape.org/) with the BiNGO plug-in, and the result of the KEGG analysis was visualized as a bar chart.

Protein-protein interaction (PPI) networks analysis. The potential targets were also input into the STRING database (http://string-db.org/) to explore the hub genes involved in 
Table I. Characteristics of the eight included studies.

\begin{tabular}{|c|c|c|c|c|c|c|}
\hline Author, year & Country & Tissue & $\begin{array}{c}\text { Sample, n } \\
\text { (HCC/control) }\end{array}$ & $\operatorname{miR}$ & Targets & (Refs.) \\
\hline Wang et al, 2017 & China & Liver cancer & $18 / 18$ & $\operatorname{miR}-449 a$ & CXCL5 & (29) \\
\hline Liu et al, 2016 & China & $\mathrm{HCC}$ & $40 / 40$ & $\operatorname{miR}-449 a$ & ADAM10 & $(30)$ \\
\hline Chen et al, 2015 & China & $\mathrm{HCC}$ & $66 / 18$ & $\operatorname{miR}-449 a$ & FOS, MET & (31) \\
\hline Liu et al, 2016 & China & Liver cancer & $48 / 48$ & $\operatorname{miR}-449 a$ & POU2F1, CAPN6 & (32) \\
\hline Zhang et al, 2016 & China & HCC cell lines & - & $\operatorname{miR}-449 a$ & CREB5 & (33) \\
\hline Buurman et al, 2012 & Germany & $\mathrm{HCC}$ & $23 / 0$ & miR-449 family & C-MET, SOX4 & $(36)$ \\
\hline Sandbothe et al, 2017 & Germany & Liver cancer & $61 / 4$ & miR-449 family & SOX4 & (24) \\
\hline Sarma et al, 2012 & America & $\begin{array}{l}\mathrm{HCV}, \mathrm{AH}, \\
\mathrm{NASH}\end{array}$ & $\begin{array}{c}10 \mathrm{HCV}, 10 \mathrm{AH} \\
10 \mathrm{NASH}, 10 \\
\text { control }\end{array}$ & $\operatorname{miR}-449 a$ & NOTCH1 & (34) \\
\hline
\end{tabular}

HCC, hepatocellular carcinoma; miR, microRNA; HCV, hepatitis C virus; AH, alcoholic hepatitis; NASH, non-alcoholic steatohepatitis.

HCC. Network nodes represented the proteins, and edges represented the protein-protein associations. All the parameter settings were selected as defaults.

Statistical analysis. The scatterplots that exhibited miR-449a expression from all the GEO datasets were generated using GraphPad Prism 5.0 (GraphPad Software, Inc., La Jolla, CA, USA). The significance of the difference between the HCC and non-cancerous liver tissues was analyzed using Student's t-tests. $\mathrm{P}<0.05$ was considered to indicate a statistically significant difference. For meta-analysis, fixed-effects models or random-effects models were used for pooling SMD depending on inconsistency statistics $\left(\mathrm{I}^{2}\right)$. When $\mathrm{I}^{2}>50 \%$, a random-effects model was selected; when $\mathrm{I}^{2}<50 \%$, a fixed-effects model was preferentially used (27). Furthermore, Begg's funnel plot was applied for calculating publication bias (28).

\section{Results}

Systematic review of original literature. With the search and screen criteria, eight studies exploring the role of miR-449a in HCC were obtained. The data from the selected studies included HCC cell lines, animal models and human biopsy specimens. However, the studies on miR-449a are currently limited and effective data could not be extracted from the articles. Thus, it was not possible to perform a meta-analysis. The characteristics of the eight included studies are presented in Table I.

The first study conducted by Wang et al (29) collected 18 liver cancer tissues along with their adjacent normal controls to explore the effect of miR-449a on liver cancer migration and invasion. This study provided preliminary evidence that miR-449a could serve as a tumor suppressor to influence the migration and invasion of liver cancer through targeting C-X-C motif chemokine 5 (CXCL5). Consistent results were also observed in four HCC cell lines.

Another study conducted by Liu et al (30) measured miR-449a expression in 40 pairs of HCC tissues and adjacent normal tissues, as well as four HCC cell lines, and demonstrated that miR-449a expression was decreased in the
HCC tissues and four cell lines. They further explored the mechanism underlying the inhibitory effects of miR-449a on the growth and metastasis of HCC cells and revealed that miR-449a functioned as a tumor suppressor miR by inhibiting the cell proliferation, colony formation, migration and invasion of HCC by partially repressing a disintegrin and metalloproteinase domain-containing protein 10 (ADAM10) expression.

Chen et al (31) investigated the miR-449a expression level in 77 HCCs and 18 normal controls. They found that the level of miR-449a in HCC was notably lower when compared to that in the normal controls. Furthermore, the portal vein tumor thrombus tissues displayed a more significant reduction of miR-449a expression, which indicated that the reduction of miR-449a in HCCs was notably associated with a more aggressive tumor phenotype. They also conducted a log-rank test, which revealed that the reduction of miR-449a was associated with short disease-free survival in patients with HCC. The study revealed that miR-449a may suppress the epithelial-mesenchymal transition (EMT) and the metastasis of HCC by inhibiting FOS and Met expression and subsequently suppressing the downstream signaling.

Another study by Liu et al (32) explored the cellular function of miR-449a in 48 cases of liver cancer. By comparison with adjacent tissues, it was revealed that miR-449a was decreased in the liver cancer tissues, and the loss of miR-449a in the liver cancer tissues was associated with tumor progression, which suggested that miR-449a may be a suppressor of cancer metastasis. The results obtained from four human liver cancer cell lines (HepG2, 7404, 7721 and 7405) were consistent with the liver tissues. Additionally, they performed a series of bioinformatics analyses and validated experiments, which revealed that miR-449a could induce $G_{1}$ arrest of the liver cancer cells, suppress cell proliferation and promote cell apoptosis. It was also identified that miR-449a affected the biological behavior of liver cancer cells by the downregulation of calpain 6 (CAPN6) and POU class 2 homeobox 1 (POU2F1).

The study conducted by Zhang et al (33) explored the molecular mechanisms of the interactions between miR-449a and HBV infection and identified that miR-449a expression was downregulated in the HCC cells. Ectopic expression of miR-449a 

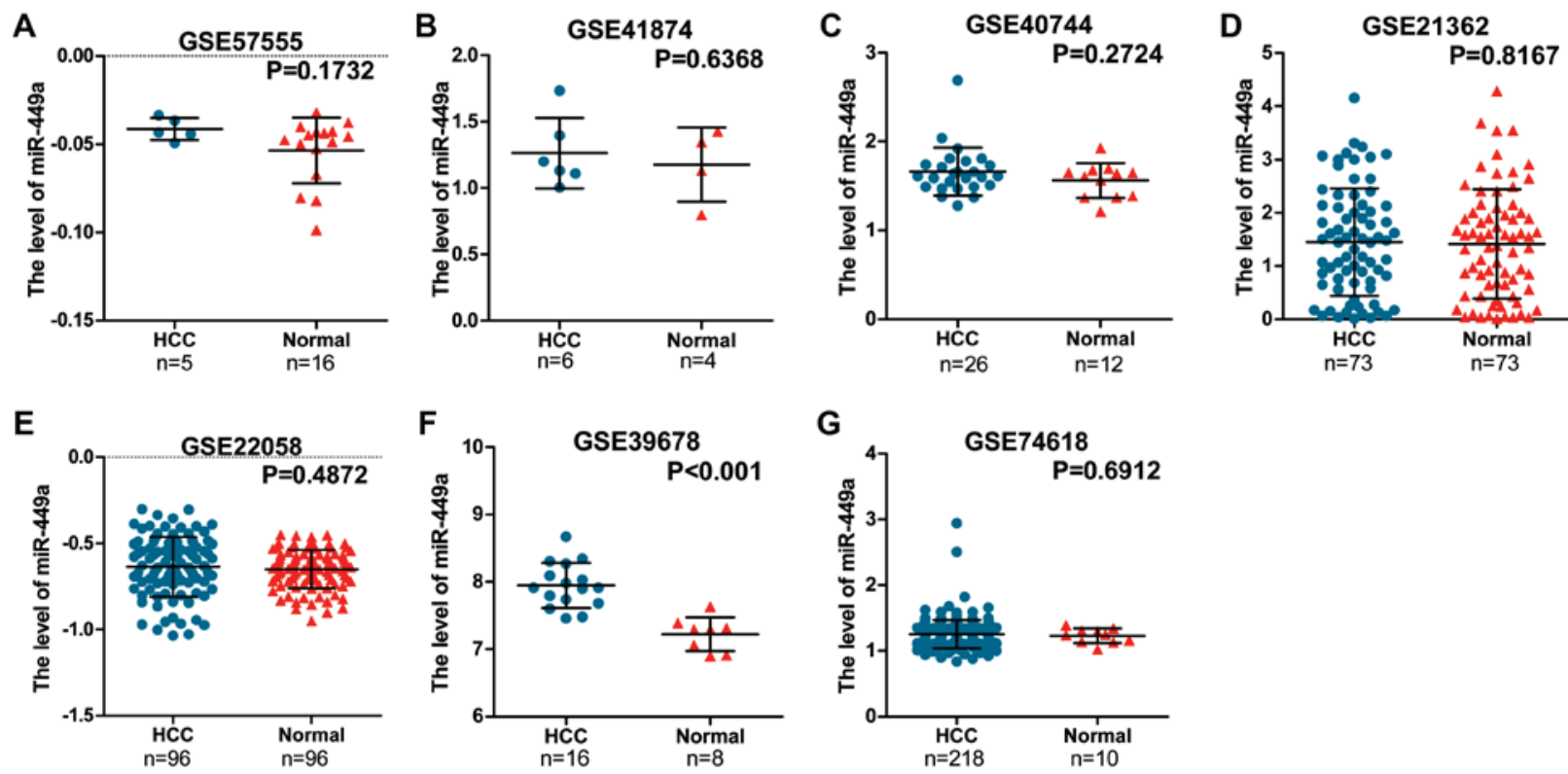

Figure 1. Scatter diagrams of miR-449a expression in seven evaluable microarray chips. (A) miR-449a expression in GSE57555, P=0.1732; (B) miR-124-3p expression in GSE41874, $\mathrm{P}=0.6368$; (C) miR-124-3p expression in GSE40744, $\mathrm{P}=0.2724$; (D) miR-124-3p expression in GSE21362, $\mathrm{P}=0.8167$; (E) miR-124-3p expression in GSE22058, $\mathrm{P}=0.4872$; (F) miR-124-3p expression in GSE39678, $\mathrm{P}<0.001$; and (G) miR-124-3p expression in GSE74618, $\mathrm{P}=0.6912$. miRNA, microRNA.

in HCC cells, to a large extent, boosted HBV replication, transcription, progeny virion secretion and antigen expression in a dose-dependent manner. They reported that miR-449a directly targeted the cyclic adenosine monophosphate (cAMP)-responsive factor and bound to cAMP responsive element binding protein 5 (CREB5), which influenced Farnesol-X-Receptor (FXR) $\alpha$ expression and facilitated HBV replication. Similarly, Sarma et al (34) analyzed the genome-wide $\mathrm{miR}$ in liver biopsies obtained from patients with chronic HCV infection and observed a downregulation of miR-449a compared with the level in the normal liver. It is well known that patients with $\mathrm{HCV}$ infection have a high risk of developing HCC (35).

Buurman et al (36) investigated 23 primary HCCs of various Gleason grades, as well as HCC cell lines and tumor xenografts, all of which demonstrated a reduced expression of miR-449a and an increased expression of c-MET in the samples. This indicated that miR-449a may function through targeting c-MET in hepatocarcinogenesis. Furthermore, analysis of the tissue samples revealed that the lowest concentrations of miR-449 were associated with high levels of c-MET, which appeared in Gleason grade 1, suggesting that the deregulation of miR-449a was mainly effective in the early stages of HCC tumorigenesis.

Recently, Sandbothe et al (24) further investigated the function of the miR-449 family using microarray analysis and public databases to identify their binding specificities, putative target genes and regulated pathways. They demonstrated that miR-449 family members significantly regulated cell cycle control, transforming growth factor (TGF) $\beta$ signaling, hepatocyte growth factor signaling and the Wnt/ $\beta$-catenin signaling pathways that were frequently altered in HCC. Following comprehensive analysis, they focused their study on the signaling of TGF- $\beta$ by targeting SOX4, which served a dual role in HCC, acting as a tumor suppressor during the early stages of liver damage, but promoting tumor progression and metastasis in advanced HCC.
Determining the expression of $\mathrm{miR}-449$ a in HCC by gathering seven GEO datasets. GEO datasets were searched to assess the expression of miR-449a between HCC and non-cancer tissues. miR-449a levels in all seven microarray chips [GSE57555 (37), GSE41874 (38), GSE40744(39), GSE21362(40), GSE22058(41), GSE39678 (42) and GSE74618 (43)] were presented in Fig. 1. Meta-analysis was conducted with seven datasets to assess the miR-449a expression level in patients with HCC in another manner, on account of the fact that the GEO microarrays could not present the role of miR-449a in HCC directly. In total, 440 patients with HCC and 219 normal controls were selected for the meta-analysis. A random-effects model was selected to calculate the pooled SMD and 95\% CI, and significant heterogeneity was identified among individual datasets $\left(\mathrm{I}^{2}=65.8 \%\right.$; $\mathrm{P}=0.007)$. The results demonstrated that a significant difference was observed between the HCC groups and the normal controls (SMD=0.40; 95\% CI, 0.01-0.79; $\mathrm{P}=0.046$ ), suggesting that the miR-449a expression levels were increased in patients with HCC rather than in the normal controls (Fig. 2).

The results of the sensitivity analysis revealed that heterogeneity may be confounded by the GSE39678 dataset (Fig. 3). The heterogeneity disappeared when the GSE39678 dataset was removed $\left(\mathrm{I}^{2}=0.0 \% ; \mathrm{P}=0.813\right)$. Hence, the $\mathrm{SMD}$ and $95 \%$ CI were pooled again as a fixed-effects model. However, no significant difference was observed in the miR-449a expression level between the patients with $\mathrm{HCC}$ and healthy controls (SMD=0.13; 95\% CI, -0.06-0.32; $\mathrm{P}=0.179$ ) (Fig. 4). The results suggested that the conclusion for the present meta-analysis was unreliable according to the inconsistent values when pooled by the separate random- and fixed-effects models.

Publication bias analysis revealed that the Begg's funnel plots (Fig. 5) were almost symmetrical, with the obtained $\operatorname{Pr}>|z|=0.072>0.05$ for miR-449a (where Pr represented the Begg's P-value and $\mathrm{z}$ represented the standard normal variate for the confidence level specified by option level), suggesting 


\begin{tabular}{|c|c|c|}
\hline Study & & $\%$ \\
\hline ID & $\operatorname{SMD}(95 \% \mathrm{CI})$ & Weight \\
\hline GSE57555 (2015) & $0.73(-0.31,1.76)$ & 9.26 \\
\hline GSE41874 (2013) & $0.32(-0.96,1.59)$ & 6.89 \\
\hline GSE40744 (2013) & $0.39(-0.30,1.08)$ & 14.42 \\
\hline GSE21362 (2011) & $0.04(-0.29,0.36)$ & 22.26 \\
\hline GSE22058 (2010) & $0.10(-0.18,0.38)$ & 23.11 \\
\hline GSE39678 (2012) & $2.35(1.25,3.45)$ & 8.53 \\
\hline GSE74618 (2016) & $0.13(-0.51,0.76)$ & 15.51 \\
\hline Overall $(\mathrm{I}-$ squared $=65.8 \%, \mathrm{P}=0.007)$ & $0.40(0.01,0.79)$ & 100.00 \\
\hline & $\mathrm{P}=0.046$ & \\
\hline NOTE: Weights are from random effects analysis & & \\
\hline
\end{tabular}

Figure 2. Forest plot of the seven evaluated datasets assessing microRNA-449a expression in HCC. A random-effects model was selected to evaluate the pooled SMD with a 95\% CI. The combined SMD suggested a marked difference between the HCC group and the normal control group (SMD=0.40; 95\% CI, 0.01-0.79; $\mathrm{P}=0.046$ ). SMD, standardized mean difference; $\mathrm{CI}$, confidence intervals; HCC, hepatocellular carcinoma.

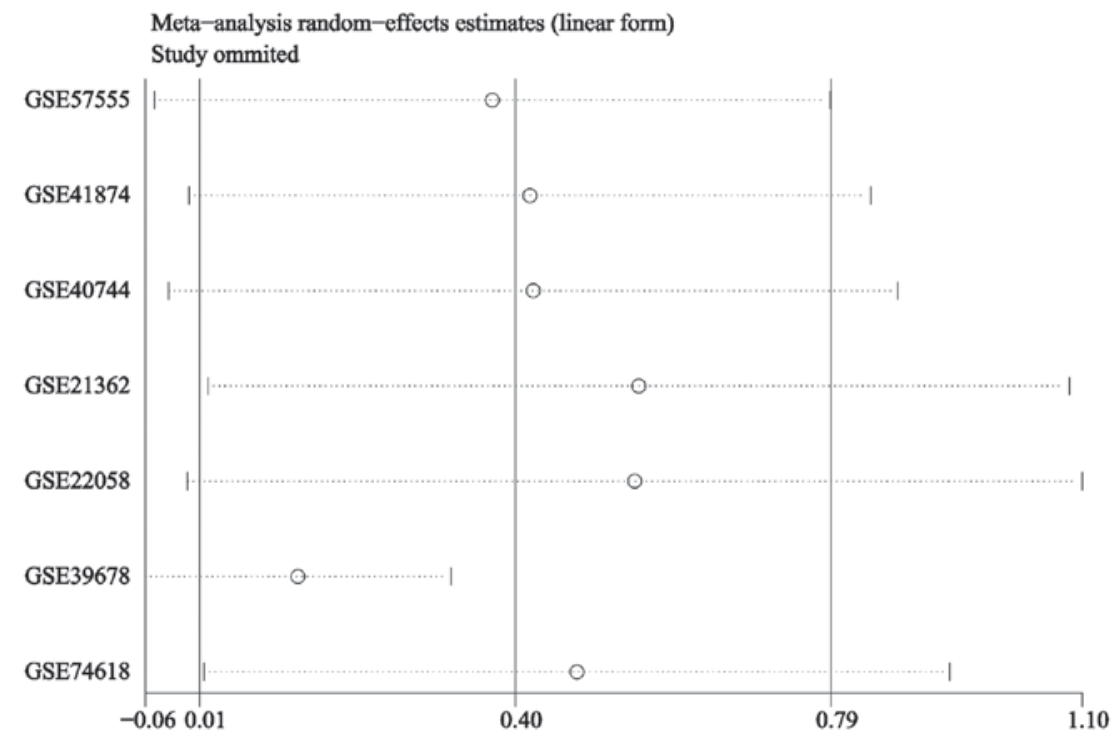

Figure 3. Sensitivity analysis of the seven evaluated datasets assessing the expression of microRNA-449a in hepatocellular carcinoma. Significant heterogeneity existed among the individual datasets $\left(\mathrm{I}^{2}=65.8 \%\right.$; $\left.\mathrm{P}=0.007\right)$, and the datasets from GSE39678 presented an obvious deviation from the estimate.

that publication bias from the included studies was absent in the present study.

Potential target genes of miR-449a in HCC. In the GSE74710 dataset, since the HCC cell line, HLE, was transfected with miR-449a mimics, the downregulated genes $(\mathrm{FC}<0.5$ and $\mathrm{P}<0.05)$ were screened as potential miR-449a target genes, and 617 eligible genes were ultimately extracted. Additionally, potential targets of miR-449a in silico were also predicted. A total of 3,662 genes that appeared in more than five databases were selected. Furthermore, a total of 1,800 HCC-related genes were recognized from NLP.

To narrow down the area and obtain more reliable miR-449a targets, three kinds of genes were overlapped. Finally, 23 genes were left for further GO and KEGG pathway analysis and PPI network analysis, which were largely representative of the prospective molecular mechanism of miR-449a in HCC (data not shown).

Potential pathways of miR-449a in HCC. The GO and KEGG enrichment analyses were performed on the potential target genes of miR-449a that were associated with HCC by the functional annotation tool of the DAVID database. The most enriched GO and KEGG terms were obtained. It was revealed that in the GO_BP category, these genes were predominantly involved in 'positive regulation of transcription from RNA polymerase II promoter' (GO: 0045944; $\mathrm{P}=1.778 \times 10^{-4}$ ), 'positive regulation of apoptotic process' (GO: 0043065; 
Study

ID
$\%$

$\operatorname{SMD}(95 \% \mathrm{Cl}) \quad$ Weight

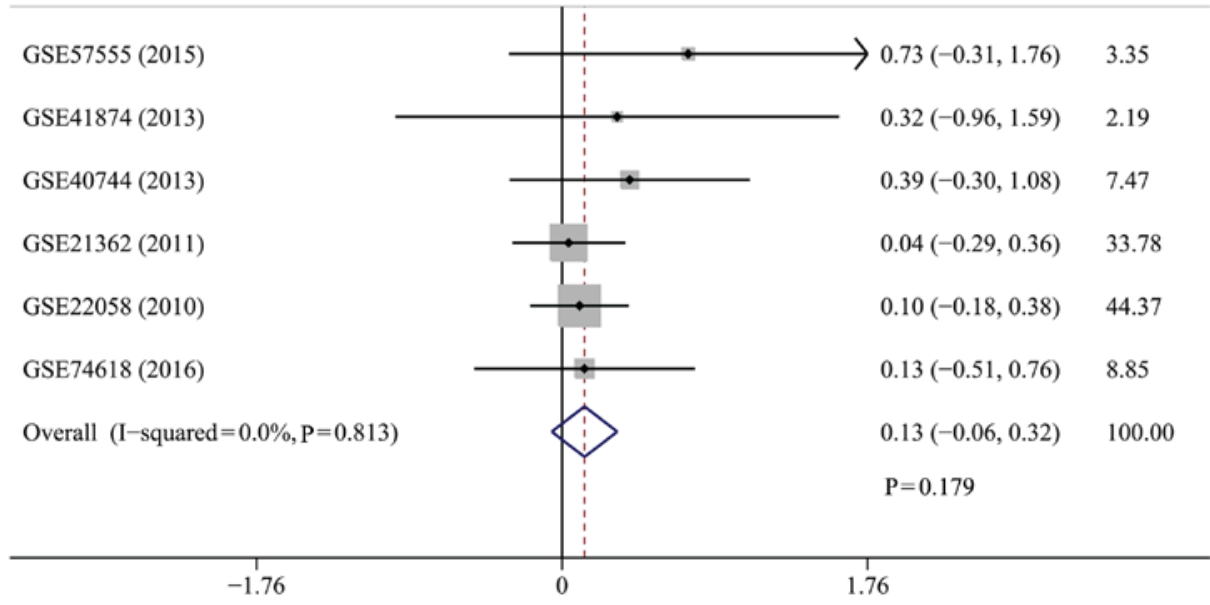

Figure 4. Forest plot of the six evaluated datasets assessing expression of microRNA-449a in hepatocellular carcinoma. A fixed-effects model was selected to evaluate the pooled SMD with a 95\% CI, and the combined SMD with a 95\% CI, following the removal of the data from GSE39678 (SMD=0.13; 95\% CI, $-0.06-0.32 ; \mathrm{P}=0.179 ; \mathrm{I}^{2}=0.0 \% ; \mathrm{P}=0.813$ ). No significant difference between the tumor and normal groups was identified. SMD, standardized mean difference; $\mathrm{CI}$, confidence interval.

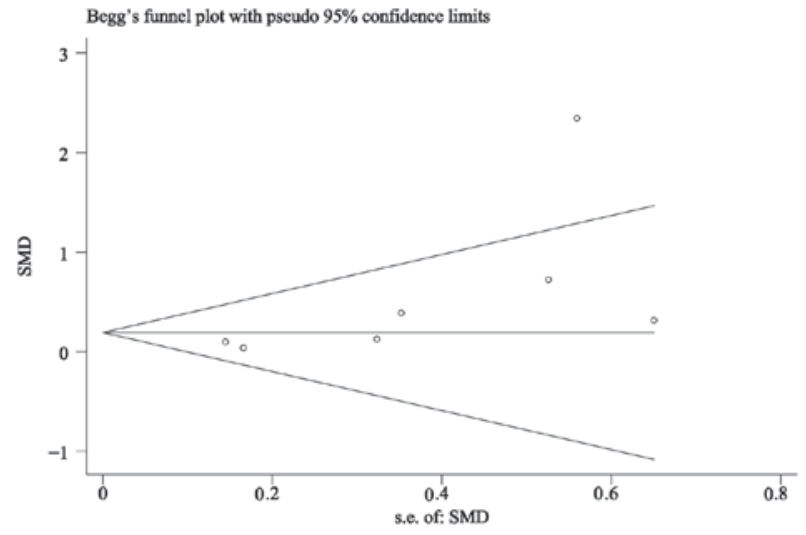

Figure 5. Begg's plot of the publication bias test of the seven studies. SMD, standardized mean difference; s.e., standard error.

$\left.\mathrm{P}=5.67 \times 10^{-4}\right)$ and 'negative regulation of cell proliferation' (GO: 0008285; $\mathrm{P}=0.0016)$. In the GO_CC category, the genes tended to affect the 'cell surface' (GO: 0009986; $\mathrm{P}=0.0265)$, 'cytoplasm' (GO: 0005737; $\mathrm{P}=0.0279$ ) and 'nucleus' (GO: 0005634; $\mathrm{P}=0.0361$ ), while in the GO_MF category, they mainly participated in 'transmembrane receptor protein tyrosine kinase activity' (GO: 0004714; $\mathrm{P}=0.0011)$, 'protein binding' (GO:0005515; $\mathrm{P}=0.0038$ ) and 'transcription factor activity, and sequence-specific DNA binding' (GO: 0003700; $\mathrm{P}=0.0069$ ). The results of the GO analysis were presented in Table II and visualized as co-expression networks using Cytoscape v3.4.0 with the BiNGO plug-in, as demonstrated in Fig. 6.

KEGG pathway investigation indicated that these target genes participated in the following categories: 'MicroRNAs in cancer' (hsa05206; $\mathrm{P}=0.0146)$, 'Cell cycle' (hsa04110; $\mathrm{P}=0.0219$ ), 'PI3K-Akt signaling pathway' (hsa04151; $\mathrm{P}=0.0243$ ) and 'N-Glycan biosynthesis' (hsa00510; $\mathrm{P}=0.0884$ ) (Table III and Fig. 7).

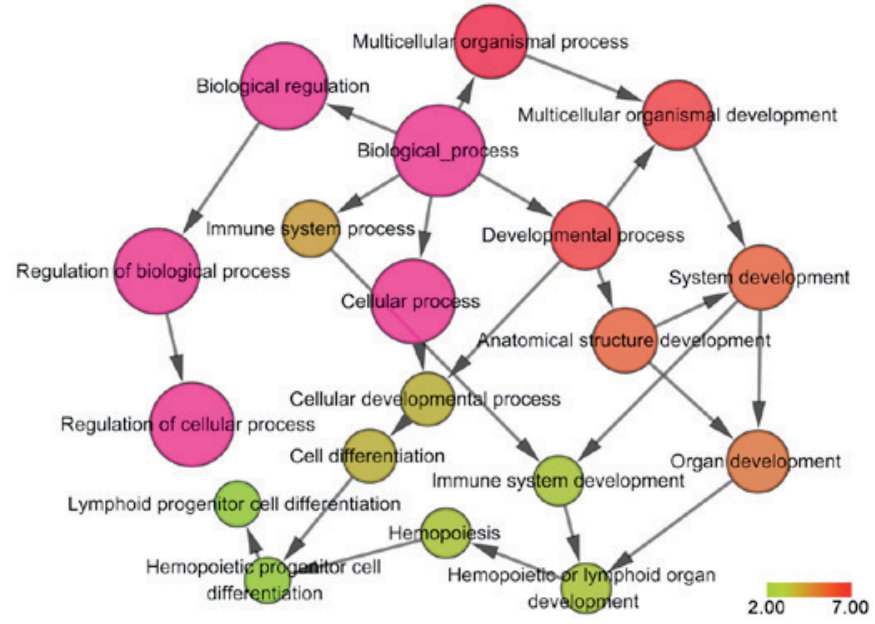

Figure 6. Gene Ontology pathway interaction maps were drawn using the Cytoscape v3.4.0 BiNGO plug-in. Color and size intensity denote the significance of the functional pathway and the enrichment of the gene. The green color represents the most significant pathway terms, and the larger size represents the more enriched genes.

In the PPI network analysis, 23 genes were input to construct an interaction network, and the PPI enrichment P-value was 0.0096, with 23 interaction edges (Fig. 8). Meanwhile, the local clustering coefficient was 0.23 . With comprehensive analysis of the network, the hub nodes, MYB, E2F5, CDK6 and CDC25A, were identified as the most essential genes in HCC, which may also function as novel targets for medical treatment.

\section{Discussion}

Accumulating evidence (44-47) has revealed that the altered expression of miR contribute to the initiation and progression of various diseases, including cancer. miR are post-transcriptional 


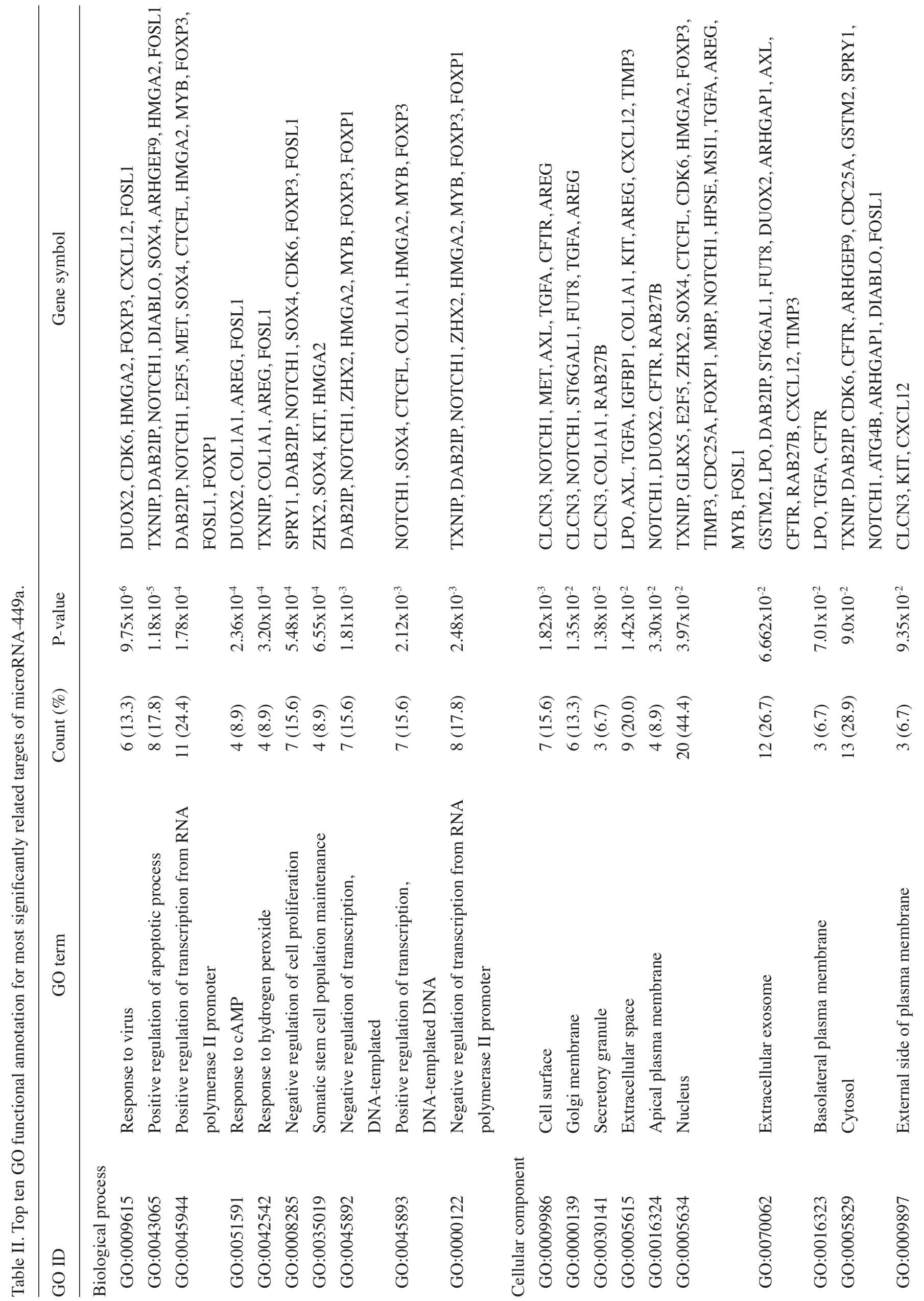




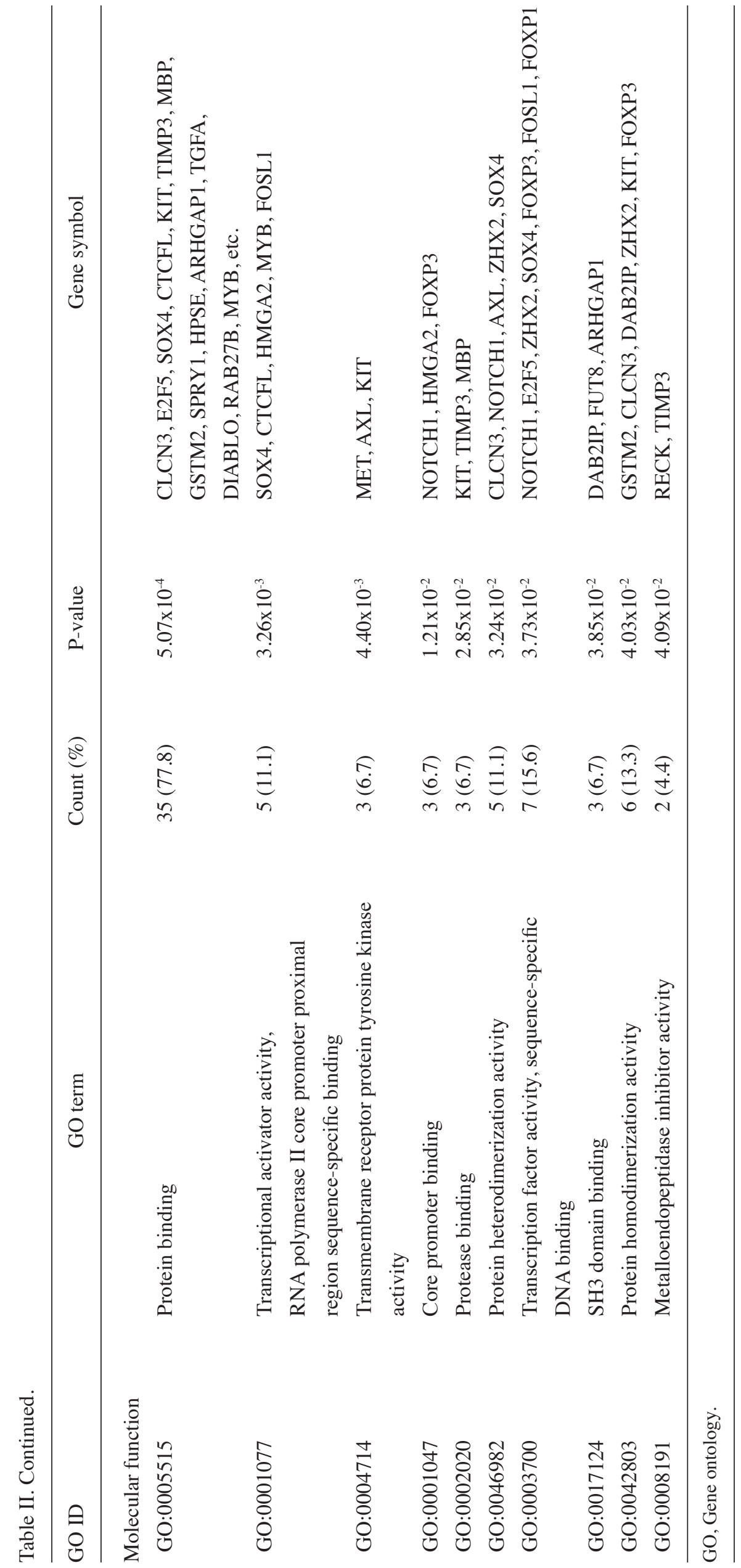


Table III. KEGG functional annotation for most significantly related targets of microRNA-449a.

\begin{tabular}{|c|c|c|c|c|}
\hline KEGG ID & KEGG term & Count (\%) & P-value & Gene symbol \\
\hline hsa05206 & MicroRNAs in cancer & $7(15.6)$ & $5.35 \times 10^{-4}$ & $\begin{array}{l}\text { RECK, NOTCH1, MET, CDK6, HMGA2, } \\
\text { TIMP3, CDC25A }\end{array}$ \\
\hline hsa02151 & PI3K-Akt signaling pathway & $5(11.1)$ & $3.83 \times 10^{-2}$ & MET, CDK6, COL1A1, KIT, MYB \\
\hline hsa05200 & Pathways in cancer & $5(11.1)$ & $5.71 \times 10^{-2}$ & MET, TGFA, CDK6, KIT, CXCL12 \\
\hline hsa04110 & Cell cycle & $3(6.7)$ & $7.85 \times 10^{-2}$ & E2F5, CDK6, CDC25A \\
\hline
\end{tabular}

KEGG, Kyoto Encyclopedia of Genes and Genomes.

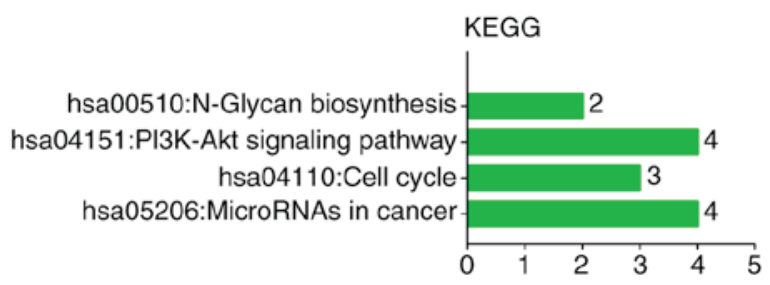

Figure 7. KEGG pathway analysis of the potential target genes of microRNA-449a in hepatocellular carcinoma. The results of the KEGG analysis were visualized as a bar chart. KEGG, Kyoto Encyclopedia of Genes and Genomes; PI3K, phosphoinositide 3 kinase.

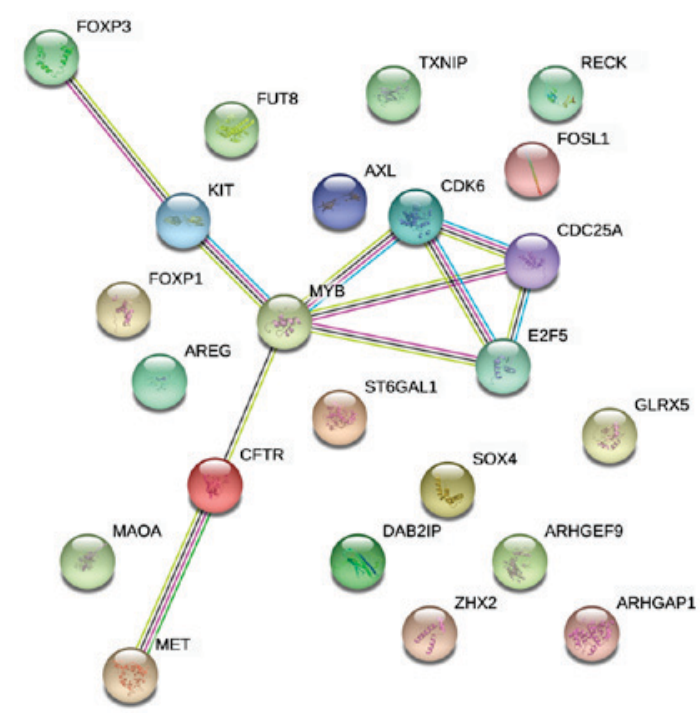

Figure 8. Protein-protein interaction networks of the potential target genes of microRNA-449a in hepatocellular carcinoma. Interactions among the 23 genes were illustrated using the STRING online database. The network nodes represent proteins and the edges represent the protein-protein associations.

regulators of gene expression and are involved in the silencing of mRNA translation (48). Investigating the altered expression of $\mathrm{miR}$ in malignancies may provide a novel and effective therapeutic target for $\mathrm{HCC}$ in the future.

The present systematic review and meta-analysis summarized eight original studies and seven microarray chips to investigate the function of miR-449a in HCC. In the original studies, all the researchers held the same viewpoint, that the miR-449a expression level was reduced in HCC. However, there were still some differences in their research. Wang et al (29) explored the effect of miR-449a on liver cancer and found that
miR-449a predominantly regulated CXCL5 expression, which encodes a chemokine that was suggested to bind the G-protein coupled receptor chemokine (C-X-C motif) receptor 2 to recruit neutrophils, promote angiogenesis and remodel connective tissues (49). The increased expression of this gene would promote cancer cell proliferation, migration and invasion. Liu et al (30) also found that miR-449a functioned as a tumor suppressor by obstructing cell proliferation, colony formation, migration and invasion of HCC by partially repressing ADAM10 expression. However, different from these two findings, Chen et al (31) documented the involvement of EMT in HCC progression. They revealed that miR-449a likely suppressed EMT and metastasis of HCC by inhibiting FOS and Met expression and suppressing the downstream signaling. The mechanism of EMT in HCC metastasis has been presented by Yang et al (50). In addition, Liu et al (32) reported that miR-449a may function by downregulating CAPN6, which is a potential oncogene in tumors and may serve a role in tumor formation by inhibiting apoptosis and promoting angiogenesis.

Another notable discovery was documented by Zhang et al (33) and Sarma et al (34). Zhang et al dissected the relationship between miR-449a and HBV infection and revealed that miR-449a acted as a linker between histone deacetylation and HBV replication, directly targeting CREB5, and CREB5 knockdown induced FXR $\alpha$ expression. FXR $\alpha$ also referred to as nuclear receptor subfamily 1 group $\mathrm{H}$ member 4 , which is a transcription factor that binds two motifs within the HBV enhancer II and the core promoter regions to promote HBV transcription and replication (51). Sarma et al (34) demonstrated that HCV downregulated miR-449a expression in human livers, which could elevate the levels of transcription factors, leading to an increase in the inflammatory response and promoting cell proliferation, which resulted in HCC.

Buurman et al (36) investigated the miR-449 family for several years, and identified at least one member (miR-449a, miR-449b or miR-449c) of the miR-449 family-targeted pathways, including Wnt/ $\beta$-catenin, p53/cell cycle control, mitogen-activated protein kinase and TGF- $\beta$. These are promising targets for HCC therapy (52). Buurman et al (36) focused on the signaling of TGF- $\beta$ and discovered that this pathway served a dual role in $\mathrm{HCC}$, acting as a tumor suppressor during early stages of liver damage, but promoting tumor progression and metastasis in advanced HCC. Furthermore, their first clinical trial for miR replacement therapy was initiated in 2013 to evaluate the safety of miR-449a in patients with unresectable primary liver cancer or other selected solid or 
hematologic malignancies (53). miR replacement therapy may provide hope for patients with liver cancer in the future.

In summary, previous original studies have shared the same views, that miR-449a is downregulated in HCC tissues and involved in various pathways by targeting multiple mRNA. However, to our surprise, the pooled results of seven microarrays revealed that miR-449a was overexpressed in HCC, which was completely contrary to the original studies. The causes of this discrepancy were analyzed, and it was speculated that microarray analysis was not sensitive enough to detect the RNA expression level compared to the method of reverse transcription-quantitative polymerase chain reaction. The majority of the microarrays gathered in the present study demonstrated no significant difference in the miR-449a expression between HCC and normal controls.

Since the original studies indicated that miR-449a may be involved in multiple signaling pathways and target multiple mRNAs, the potential targets of miR-449a in HCC were overlapped to explore whether miR-449a may exert its function in other pathways. The potential targets, including GEO downregulated genes, in silico predicted genes, as well as HCC-related genes recognized from NLP, were merged with three different resources to obtain more reliable targets. However, limitations remained in the present study. The GEO downregulated genes were required to have an $\mathrm{FC}$ value of $<0.5$ and a $\mathrm{P}$-value of $<0.05$ for inclusion, and the adjusted P-value was also taken into consideration, which provides a more accurate indication of the level of false positives for a given cut-off value. However, the adjusted P-values of all the eligible genes were distributed from $0.1-0.3$. The results revealed that more false positives may exist in these included genes. Therefore, the miRWalk database, which contains 12 prediction algorithms, and NLP were used to identify more reliable targets. The present results revealed that, in addition to 'cell cycle pathway' (hsa04110), miR-449a may participate in 'MicroRNAs in cancer' (hsa05206), 'PI3K-Akt signaling pathway' (hsa04151) and 'N-Glycan biosynthesis pathways' (hsa00510) to regulate HCC tumorigenesis.

In addition, PPI network analysis was conducted, and hub nodes, including MYB, E2F5, CDK6 and CDC25A, were identified as the most essential genes in HCC. Among them, MYB encoded a protein with three helix-turn-helix DNA-binding domains, which functioned as transcription regulators (54). It was reported that MYB expression was increased at the mRNA level in HCC (55). E2F5 is a member of the E2F transcription factor family and binds to the promoters of target genes involved in cell cycle control, and consequently modulates the expression of these targets (56). The members of the E2F family have been divided into the activator (E2F1-E2F3) and repressor (E2F4-E2F8) subclasses (57). The overexpression or amplification of the E2F5 gene has been reported in various solid tumors, such as HCC. For example, Jiang et al (58) provided evidence that E2F5 was commonly upregulated in HCC and E2F5 knockdown notably inhibited the growth of HCC cells. Whether MYB or E2F5 deregulation in HCC is targeted by miR-449a requires further investigation. The phosphorylation of CDKs is required for timely progression through the cell division cycle (59). CDK6 has been demonstrated to phosphorylate and regulate the activity of the tumor suppressor protein, retinoblastoma $(60,61)$. Altered expression of this gene has been observed in multiple human cancer types (62-64). Likewise, the dysregulation of CDK6 in $\mathrm{HCC}$ has also been previously reported $(65,66)$. CDC25A, a member of the CDC25 family of phosphatases, is a cell cycle and apoptotic regulator (67). Previous research has reported that dephosphorylation of CDC25 is the rate-limiting step for CDK activation. Their phosphorylation removes the inhibitory phosphorylation on the CDK and regulates the $\mathrm{G}_{1}-\mathrm{S}$ phase progression in the cell cycle (68).

In conclusion, as the number of studies in the present systematic review was small and these studies provided limited data to conduct a meta-analysis, it is difficult to deduce any definite conclusion. Based on the evidence presented here, miR-449a was revealed to be reduced in HCC and is likely to be involved in various kinds of signaling pathways that target multiple mRNAs to exert its function in HCC. Larger samples and further investigation are required to validate this conclusion.

\section{Competing interests}

The authors declare that they have no competing interests.

\section{References}

1. Wan LC, Wang F, Guo X, Lu S, Qiu Z, Zhao Y, Zhang H and Lin J: Identification and characterization of small non-coding RNAs from Chinese fir by high throughput sequencing. BMC Plant Biol 12: 146, 2012.

2. Bussotti G, Notredame C and Enright AJ: Detecting and comparing non-coding RNAs in the high-throughput era. Int J Mol Sci 14: 15423-15458, 2013.

3. Iyer MK, Niknafs YS, Malik R, Singhal U, Sahu A, Hosono Y, Barrette TR, Prensner JR, Evans JR, Zhao S, et al: The landscape of long noncoding RNAs in the human transcriptome. Nat Genet 47: 199-208, 2015.

4. Wang H, Ke C, Ma X, Zhao Q, Yang M, Zhang W and Wang J: MicroRNA-92 promotes invasion and chemoresistance by targeting GSK $3 \beta$ and activating Wnt signaling in bladder cancer cells. Tumour Biol 37: 16295-16304, 2016.

5. Cech TR and Steitz JA: The noncoding RNA revolution-trashing old rules to forge new ones. Cell 157: 77-94, 2014.

6. Dhamija S and Diederichs S: From junk to master regulators of invasion: lncRNA functions in migration, EMT and metastasis. Int J Cancer 139: 269-280, 2016.

7. Wu W, Liu S, Liang Y, Zhou Z and Liu X: MiR-7 inhibits progression of hepatocarcinoma by targeting KLF-4 and promises a novel diagnostic biomarker. Cancer Cell Int 17: 31, 2017.

8. Morey $\mathrm{C}$ and Avner P: Employment opportunities for non-coding RNAs. FEBS Lett 567: 27-34, 2004.

9. Calin GA and Croce CM: MicroRNA signatures in human cancers. Nat Rev Cancer 6: 857-866, 2006.

10. Ling H, Fabbri $M$ and Calin GA: MicroRNAs and other non-coding RNAs as targets for anticancer drug development. Nat Rev Drug Discov 12: 847-865, 2013.

11. Dehury B, Panda D, Sahu J, Sahu M, Sarma K, Barooah M, Sen $\mathrm{P}$ and Modi M: In silico identification and characterization of conserved miRNAs and their target genes in sweet potato (Ipomoea batatas L.) expressed sequence tags (ESTs). Plant Signal Behav 8: e26543, 2013.

12. Cong D, He M, Chen S, Liu X, Liu X and Sun H: Expression profiles of pivotal microRNAs and targets in thyroid papillary carcinoma: An analysis of The Cancer Genome Atlas. Onco Targets Ther 8: 2271-2277, 2015.

13. Polioudakis D, Abell NS and Iyer VR: MiR-191 regulates primary human fibroblast proliferation and directly targets multiple oncogenes. PLoS One 10: e0126535, 2015.

14. Yuan S, Tang C, Zhang Y, Wu J, Bao J, Zheng H, Xu C and Yan W: $\mathrm{mir}-34 \mathrm{~b} / \mathrm{c}$ and $\mathrm{mir}-449 \mathrm{a} / \mathrm{b} / \mathrm{c}$ are required for spermatogenesis, but not for the first cleavage division in mice. Biol Open 4: 212-223, 2015.

15. Kumar P, Sharad S, Petrovics G, Mohamed A, Dobi A, Sreenath TL, Srivastava S and Biswas R: Loss of miR-449a in ERG-associated prostate cancer promotes the invasive phenotype by inducing SIRT1. Oncotarget 7: 22791-22806, 2016. 
16. Shi J, Liu Y, Liu J and Zhou J: Hsa-miR-449a genetic variant is associated with risk of gastric cancer in a Chinese population. Int J Clin Exp Pathol 8: 13387-13392, 2015.

17. You J, Zhang Y, Li Y, Fang N, Liu B, Zu L and Zhou Q: MiR-449a suppresses cell invasion by inhibiting MAP2K1 in non-small cell lung cancer. Am J Cancer Res 5: 2730-2744, 2015.

18. Torre LA, Bray F, Siegel RL, Ferlay J, Lortet-Tieulent J and Jemal A: Global cancer statistics, 2012. CA Cancer J Clin 65: 87-108, 2015

19. Guo P, Huang ZL, Yu P and Li K: Trends in cancer mortality in China: An update. Ann Oncol 23: 2755-2762, 2012.

20. Center MM and Jemal A: International trends in liver cancer incidence rates. Cancer Epidemiol Biomarkers Prev 20: 2362-2368, 2011.

21. Chen CF, Hsu EC, Lin KT, Tu PH, Chang HW, Lin CH, Chen YJ, Gu DL, Lin CH, Wu JY, et al: Overlapping high-resolution copy number alterations in cancer genomes identified putative cancer genes in hepatocellular carcinoma. Hepatology 52: 1690-1701, 2010.

22. Takeshima N, Sozu T, Tajika A, Ogawa Y, Hayasaka Y and Furukawa TA: Which is more generalizable, powerful and interpretable in meta-analyses, mean difference or standardized mean difference? BMC Med Res Methodol 14: 30, 2014

23. ValeraEM,Faraone SV,Murray KE and Seidman LJ: Meta-analysis of structural imaging findings in attention-deficit/hyperactivity disorder. Biol Psychiatry 61: 1361-1369, 2007.

24. Sandbothe M, Buurman R, Reich N, Greiwe L, Vajen B, Gürlevik E, Schäffer V, Eilers M, Kühnel F, Vaquero A, et al: The microRNA-449 family inhibits TGF- $\beta$-mediated liver cancer cell migration by targeting SOX4. J Hepatol 66: 1012-1021, 2017.

25. Dweep H and Gretz N: miRWalk2.0: A comprehensive atlas of microRNA-target interactions. Nat Methods 12: 697, 2015.

26. Zhang X, Tang W, Chen G, Ren F, Liang H, Dang Y and Rong M: An encapsulation of gene signatures for hepatocellular carcinoma, MicroRNA-132 predicted target genes and the corresponding overlaps. PLoS One 11: e0159498, 2016.

27. Lau J, Ioannidis JP and Schmid CH: Quantitative synthesis in systematic reviews. Ann Intern Med 127: 820-826, 1997.

28. Begg CB and Mazumdar M: Operating characteristics of a rank correlation test for publication bias. Biometrics 50: 1088-1101, 1994.

29. Wang Q, Huang CS, Yu W, et al: MicroRNA-449a suppresses liver cancer migration and invasion through targeting CXC chemokine ligand 5. Chin J Exp Surg 34: 228-230, 2017.

30. Liu S, Liu K, Zhang W, Wang Y, Jin Z, Jia B and Liu Y: miR-449a inhibits proliferation and invasion by regulating ADAM10 in hepatocellular carcinoma. Am J Transl Res 8: 2609-2619, 2016.

31. Chen SP, Liu BX, Xu J, Pei XF, Liao YJ, Yuan F and Zheng F: MiR-449a suppresses the epithelial-mesenchymal transition and metastasis of hepatocellular carcinoma by multiple targets. BMC Cancer 15: 706, 2015.

32. Liu Y, Wang Y, Sun X, Mei C, Wang L, Li Z and Zha X: miR-449a promotes liver cancer cell apoptosis by downregulation of Calpain 6 and POU2F1. Oncotarget 7: 13491-13501, 2016

33. Zhang X, Liu H, Xie Z, Deng W, Wu C, Qin B, Hou J and Lu M: Epigenetically regulated miR-449a enhances hepatitis B virus replication by targeting cAMP-responsive element binding protein 5 and modulating hepatocytes phenotype. Sci Rep 6: 25389, 2016.

34. Sarma NJ, Tiriveedhi V, Subramanian V, Shenoy S, Crippin JS, Chapman WC and Mohanakumar T: Hepatitis C virus mediated changes in miRNA-449a modulates inflammatory biomarker YKL40 through components of the NOTCH signaling pathway. PLoS One 7: e50826, 2012.

35. El-Serag HB, Kramer J, Duan Z and Kanwal F: Racial differences in the progression to cirrhosis and hepatocellular carcinoma in HCV-infected veterans. Am J Gastroenterol 109: 1427-1435, 2014.

36. Buurman R, Gürlevik E, Schäffer V, Eilers M, Sandbothe M, Kreipe H, Wilkens L, Schlegelberger B, Kühnel F and Skawran B: Histone deacetylases activate hepatocyte growth factor signaling by repressing microRNA-449 in hepatocellular carcinoma cells. Gastroenterology 143: 811-820.e15, 2012.

37. Murakami Y, Kubo S, Tamori A, Itami S, Kawamura E, Iwaisako K, Ikeda K, Kawada N, Ochiya T and Taguchi YH: Comprehensive analysis of transcriptome and metabolome analysis in intrahepatic cholangiocarcinoma and hepatocellular carcinoma. Sci Rep 5: 16294, 2015.

38. Morita K, Shirabe K, Taketomi A, Soejima Y, Yoshizumi T, Uchiyama H, Ikegami T, Yamashita Y, Sugimachi K, Harimoto N, et al: Relevance of microRNA-18a and microRNA-199a-5p to hepatocellular carcinoma recurrence after living donor liver transplantation. Liver Transpl 22: 665-676, 2016
39. Diaz G, Melis M, Tice A, Kleiner DE, Mishra L, Zamboni F and Farci P: Identification of microRNAs specifically expressed in hepatitis $\mathrm{C}$ virus-associated hepatocellular carcinoma. Int J Cancer 133: 816-824, 2013

40. Sato F, Hatano E, Kitamura K, Myomoto A, Fujiwara T, Takizawa S, Tsuchiya S, Tsujimoto G, Uemoto $S$ and Shimizu K: MicroRNA profile predicts recurrence after resection in patients with hepatocellular carcinoma within the Milan Criteria. PLoS One 6: e16435, 2011.

41. Liu AM, Yao TJ, Wang W, Wong KF, Lee NP, Fan ST, Poon RT, Gao C and Luk JM: Circulating miR-15b and miR-130b in serum as potential markers for detecting hepatocellular carcinoma: A retrospective cohort study. BMJ Open 2: e000825, 2012.

42. Noh JH, Chang YG, Kim MG, Jung KH, Kim JK, Bae HJ, Eun JW, Shen Q, Kim SJ, Kwon SH, et al: MiR-145 functions as a tumor suppressor by directly targeting histone deacetylase 2 in liver cancer. Cancer Lett 335: 455-462, 2013.

43. Martinez-Quetglas I, Pinyol R, Dauch D, Torrecilla S, Tovar V, Moeini A, Alsinet C, Portela A, Rodriguez-Carunchio L, Solé M, et al: IGF2 Is Up-regulated by epigenetic mechanisms in hepatocellular carcinomas and is an actionable oncogene product in experimental models. Gastroenterology 151: 1192-1205, 2016

44. Petrovic N, Ergün S and Isenovic ER: Levels of MicroRNA heterogeneity in cancer biology. Mol Diagn Ther 21: 511-523, 2017.

45. Luo LJ, Zhang LP, Duan CY, Wang B, He NN, Abulimiti P and Lin Y: The inhibition role of miR-22 in hepatocellular carcinoma cell migration and invasion via targeting CD147. Cancer Cell Int 17: 17, 2017

46. Xia W, Zhou J, Luo H, Liu Y, Peng C, Zheng W and Ma W: MicroRNA-32 promotes cell proliferation, migration and suppresses apoptosis in breast cancer cells by targeting FBXW7. Cancer Cell Int 17: 14, 2017.

47. Liu C, Li G, Yang N, Su Z, Zhang S, Deng T, Ren S, Lu S Tian Y, Liu Y and Qiu Y: miR-324-3p suppresses migration and invasion by targeting WNT2B in nasopharyngeal carcinoma. Cancer Cell International 17: 2, 2017.

48. Towler BP, Jones CI and Newbury SF: Mechanisms of regulation of mature miRNAs. Biochem Soc Trans 43: 1208-1214, 2015

49. Tacke F, Zimmermann HW, Trautwein C and Schnabl B: CXCL5 plasma levels decrease in patients with chronic liver disease. J Gastroenterol Hepatol 26: 523-529, 2011

50. Yang MH, Chen CL, Chau GY, Chiou SH, Su CW, Chou TY, Peng WL and Wu JC: Comprehensive analysis of the independent effect of twist and snail in promoting metastasis of hepatocellular carcinoma. Hepatology 50: 1464-1474, 2009.

51. Curtil C, Enache LS, Radreau P, Dron AG, Scholtès C, Deloire A, Roche D, Lotteau V, André P and Ramière C: The metabolic sensors FXR $\alpha$, PGC-1 $\alpha$, and SIRT1 cooperatively regulate hepatitis B virus transcription. FASEB J 28: 1454-1463, 2014.

52. Schulze K, Imbeaud S, Letouze E, Alexandrov LB, Calderaro J, Rebouissou S, Couchy G, Meiller C, Shinde J, Soysouvanh F, et al: Exome sequencing of hepatocellular carcinomas identifies new mutational signatures and potential therapeutic targets. Nat Genet 47: 505-511, 2015

53. ClinicalTrials.gov: A multicenter phase I study of MRX34, microRNA miR-RX34 liposomal injection, NCT01829971.2013.

54. Veals SA, Schindler C, Leonard D, Fu XY, Aebersold R, Darnell JE Jr and Levy DE: Subunit of an alpha-interferon-responsive transcription factor is related to interferon regulatory factor and Myb families of DNA-binding proteins. Mol Cell Biol 12: 3315-3324, 1992

55. Yang H, Huang ZZ, Wang J and Lu SC: The role of c-Myb and $\mathrm{Sp} 1$ in the up-regulation of methionine adenosyltransferase $2 \mathrm{~A}$ gene expression in human hepatocellular carcinoma. FASEB J 15: 1507-1516, 2001 .

56. Wan Z, Zhi N, Wong S, Keyvanfar K, Liu D, Raghavachari N, Munson PJ, Su S, Malide D, Kajigaya S and Young NS: Human parvovirus B19 causes cell cycle arrest of human erythroid progenitors via deregulation of the E2F family of transcription factors. J Clin Invest 120: 3530-3544, 2010

57. Chen HZ, Tsai SY and Leone G: Emerging roles of E2Fs in cancer: An exit from cell cycle control. Nat Rev Cancer 9: 785-797, 2009.

58. Jiang Y, Yim SH, Xu HD, Jung SH, Yang SY, Hu HJ, Jung CK and Chung YJ: A potential oncogenic role of the commonly observed E2F5 overexpression in hepatocellular carcinoma. World J Gastroenterol 17: 470-477, 2011.

59. Suryadinata R, Sadowski M and Sarcevic B: Control of cell cycle progression by phosphorylation of cyclin-dependent kinase (CDK) substrates. Biosci Rep 30: 243-255, 2010. 
60. Iyirhiaro GO, Im DS, Boonying W, Callaghan SM, During MJ, Slack RS and Park DS: Cdc25A Is a critical mediator of ischemic neuronal death in vitro and in vivo. J Neurosci 37: 6729-6740, 2017.

61. Shi Y, Qian ZR, Zhang S, Li W, Masugi Y, Li T, Chan JA, Yang J, Da Silva A, Gu M, et al: Cell cycle protein expression in neuroendocrine tumors: Association of CDK4/CDK6, CCND1, and phosphorylated retinoblastoma protein with proliferative index. Pancreas 46: 1347-1353, 2017.

62. Shang A, Lu WY, Yang M, Zhou C, Zhang H, Cai ZX, Wang WW, Wang WX and Wu GQ: miR-9 induces cell arrest and apoptosis of oral squamous cell carcinoma via CDK 4/6 pathway. Artif Cells Nanomed Biotechnol: 1-9, 2017.

63. Lulla AR, Slifker MJ, Zhou Y, Lev A, Einarson MB, Dicker DT and El-Deiry WS: miR-6883 family miRNAs target CDK4/6 to induce G1 phase cell cycle arrest in colon cancer cells. Cancer Res 77: 6902-6913, 2017.

64. Dall'Acqua A, Sonego M, Pellizzari I, Pellarin I, Canzonieri V, D'Andrea S, Benevol S, Sorio R, Giorda G, Califano D, et al: CDK6 protects epithelial ovarian cancer from platinum-induced death via FOXO3 regulation. EMBO Mol Med 9: 1415-1433, 2017.

65. Zhu H, Wang G, Zhou X, Song X, Gao H, Ma C, Chang H, Li H, Liu FF, Lu J and Ma J: miR-1299 suppresses cell proliferation of hepatocellular carcinoma (HCC) by targeting CDK6. Biomed Pharmacother 83: 792-797, 2016.
66. Wu H, Tao J, Li X, Zhang T, Zhao L, Wang Y, Zhang L, Xiong J, Zeng Z, Zhan N, et al: MicroRNA-206 prevents the pathogenesis of hepatocellular carcinoma by modulating expression of met proto-oncogene and cyclin-dependent kinase 6 in mice. Hepatology 66: 1952-1967, 2017.

67. Zou X, Tsutsui T, Ray D, Blomquist JF, Ichijo H, Ucker DS and Kiyokawa H: The cell cycle-regulatory CDC25A phosphatase inhibits apoptosis signal-regulating kinase 1. Mol Cell Biol 21: 4818-4828, 2001.

68. Sur S and Agrawal DK: Phosphatases and kinases regulating CDC25 activity in the cell cycle: Clinical implications of CDC25 overexpression and potential treatment strategies. Mol Cell Biochem 416: 33-46, 2016.

This work is licensed under a Creative Commons Attribution-NonCommercial-NoDerivatives 4.0 International (CC BY-NC-ND 4.0) License. 